\title{
Leading Twist Parton Distribution Amplitudes in Heavy Vector Mesons
}

\author{
Fei Gao ${ }^{1,2, a}$, Minghui Ding ${ }^{1,2, b}$, Lei Chang ${ }^{3, ~ c}$, Yu-Xin Liu ${ }^{1,2,4, d}$, and Craig D. Roberts ${ }^{5, e}$ \\ ${ }^{1}$ Department of Physics and State Key Laboratory of Nuclear Physics and Technology, Peking University, \\ Beijing 100871, China \\ ${ }^{2}$ Collaborative Innovation Center of Quantum Matter, Beijing 100871, China \\ ${ }^{3}$ CSSM, School of Chemistry and Physics, University of Adelaide, Adelaide SA 5005, Australia \\ ${ }^{4}$ Center for High Energy Physics, Peking University, Beijing 100871, China \\ ${ }^{5}$ Division of Physics, Argonne National Laboratory, Argonne, IL 60439, USA
}

\begin{abstract}
We employed QCD's Dyson-Schwinger equations (DSEs) for heavy quarks and obtained the leading twist parton distribution amplitudes (PDAs) in heavy vector mesons $J / \Psi$ and $\Upsilon$. We found that all of the amplitudes are narrower than the asymptotic form, while they deviate from $\delta$ function. This indicates that the interaction between the two continent quarks are still important in the mesons consisted of charm and bottom quarks.
\end{abstract}

\section{Introduction}

The leading twist parton distribution amplitudes (PDAs) of mesons are defined by integrating out the transeverse momentum $k_{\perp}$ from the light front wave function [1]. They play an essential role in the hard exclusive processes [2-5], and moreover, the vector meson PDAs can be inferred [6, 7] from diffractive vector-meson production experiments at the Hadron Electron Ring Accelerator (HERA) [8-10]. Herein we calculate the leading twist PDAs in heavy vector mesons within DSEs approach.

The DSEs approach has been employed to calculate the light front information including the light flavour parton distribution amplitudes [11-13], parton distribution functions [14] and pion form factor [15] through projecting the Bethe-Salpeter wave function onto the light front. It is then natural to generalize the calculation to the heavy quarks case to obtain the PDAs for vector mesons $J / \Psi$ and $\Upsilon$. Our results show that for the mesons consisted of the charm and bottom quarks, their PDAs still deviate from $\delta$ function. Such heavy meson's PDAs will bring in the non-factorizable corrections in the hard exclusive processes [4].

Our presentation is organized as follows. In Sec. 2 we introduce some relevant definitions to calculate mesons' PDAs. In Sec. 3 we give our results of PDAs and some discussions.

\footnotetext{
a. e-mail: hiei@pku.edu.cn,speaker

b. e-mail: mhding@pku.edu.cn

c. e-mail: lei.chiong@gmail.com

d. e-mail: yxliu@pku.edu.cn

e. e-mail: c.d.roberts@anl.gov
} 


\section{PDAs and Bethe-Salpeter wave function}

The leading twist PDAs could be defined as the following projection of Bethe-Salpeter wave function onto the light front $[11,12]$, at leading twist there are two independent PDAs for vector meson. They could be defined as following.

$$
\begin{aligned}
& f_{V}^{\perp} \phi_{V \perp}(x, \zeta) m_{V}^{2}=n \cdot \operatorname{Ptr}_{C D} Z_{T}(\zeta, \Lambda) \int_{d q}^{\Lambda} \delta\left(n \cdot q_{+}-x n \cdot P\right) \sigma_{\mu \lambda} P_{\mu} \chi_{\lambda}(q ; P), \\
& f_{V} n \cdot P \phi_{V \|}(x, \zeta)=m_{V} t_{C D} Z_{2}(\zeta, \Lambda) \int_{d q}^{\Lambda} \delta\left(n \cdot q_{+}-x n \cdot P\right) n \cdot \gamma n_{\lambda} \chi_{\lambda}(q ; P),
\end{aligned}
$$

where the trace is over the color and spinor indices; $\mathrm{V}$ is for vector meson; $\perp$ is for the transversal PDA of vector meson and $\|$ is for the longitudinal part; $Z_{2, T}(\zeta, \Lambda)$ is the quark wave function renormalization constant, with $\zeta$ the renormalization scale; $n$ is a lightlike four-vector; $P$ is the meson's four-momentum, with $P^{2}=-m_{V}^{2}$ and $n \cdot P=-m_{V}$, with $m_{V}$ being the meson's mass; $f_{V}^{\perp}$ and $f_{V}$ are decay constants which would normalize the distributions as $\int_{0}^{1} d x \phi_{V \perp, V \|}(x)=1$. The meson's Bethe-Salpeter wave function is defined as $\chi_{\lambda}(q ; P)=S\left(q_{+}\right) \Gamma_{\lambda}(q, P) S\left(q_{-}\right)$with $\Gamma_{\lambda}$ the Bethe-Salpeter amplitude, $S$ the dressed quark propagator and $q_{+}=q+\eta P, q_{-}=q-(1-\eta) P, \eta \in[0,1]$. Using Eq. (1a-1b) and the numerical results of meson's Bethe-Salpeter amplitudes, one may get the moments of distribution $\left.<x^{m}\right\rangle=\int_{0}^{1} d x x^{m} \phi(x)$, and then would fit the PDAs.

The PDAs in heavy flavour mesons are quite different from the light quark case. The PDA would tend to be $\delta$ function as the quark mass goes to infinity, and hence intuitively, it would be appropriate to fit the PDAs in heavy flavour mesons through an exponential distribution instead of the Gegenbauer polynomials used in the light quark case $[1,11,12,16]$. Here we use the following form :

$$
\phi(x)=6 a x(1-x) e^{-c\left(\frac{3}{2}-6 x(1-x)\right)} .
$$

\section{Results and Discussions}

\subsection{Meson properties in rainbow-ladder truncation}

In our calculation, we solve the quark gap equation and Bethe-Salpeter equation obtained with the rainbow-ladder truncation of QCD's DSEs [17-19] and the interaction introduced in Ref. [20]. We employ two choices for the parameters in this interaction, $D \omega=(0.87 \mathrm{GeV})^{3}$ and $D \omega=(0.55 \mathrm{GeV})^{3}$. The current charm and bottom quark masses are chosen to be $m_{c}\left(\zeta=m_{c}\right)=1.29 \mathrm{GeV}$ and $m_{b}(\zeta=$ $\left.m_{b}\right)=4.16 \mathrm{GeV}$ which are renormalized at the respective current mass scale. After then we get the

\begin{tabular}{|c|c|c|c|c|c|c|}
\hline & $m_{J / \Psi}$ & $m_{\Upsilon}$ & $f_{J / \Psi}$ & $f_{J / \Psi}^{\perp}$ & $f_{\Upsilon}$ & $f_{\Upsilon}^{\perp}$ \\
\hline$D \omega=(0.87 \mathrm{GeV})^{3}$ & 3.17 & 9.46 & 0.483 & 0.386 & 0.602 & 0.506 \\
\hline$D \omega=(0.55 \mathrm{GeV})^{3}$ & 3.17 & 9.63 & 0.288 & 0.226 & 0.496 & 0.440 \\
\hline
\end{tabular}
meson properties including the masses and decay constants exhibited in Table. 1.

Tableau 1. Mesons mass and decay constants $(\mathrm{GeV})$

The results does not change a lot when we vary the parameters from $D \omega=(0.87 \mathrm{GeV})^{3}$ to $D \omega=$ $(0.55 \mathrm{GeV})^{3}$. As the interaction strength becomes smaller, the decay constants decrease. Besides, the gap between $f^{\perp}$ and $f$ becomes smaller, and these two decay constants will gradually become identical as in leading order approximation [21]. Besides, if we employ a naive quark model, the two decay 
constants for vector meson satisfy the relation [22] : $2 m_{q}(\zeta) f_{V}=m_{V} f_{V}^{\perp}$, and thus the deviation for this relation indicates the dynamical chiral symmetry breaking. For light flavour mesons, this relation is sabotaged severely owing to the disparity between the large dynamical quark mass and the current quark mass, while for heavy flavour vector mesons, this relation is almost satisfied. This reveals that the effect of dynamical chiral symmetry breaking (DCSB) becomes less superior since the current quark mass is quite large compared to the dynamical mass from DCSB for heavy vector mesons and hence will lead to quite different behaviour compared to the light mesons.

\subsection{Parton Distribution Amplitudes}

In light quark case, we cannot handle the calculation for the moments in Euclidean space since the $\left(\frac{n \cdot q_{+}}{n \cdot P}\right)$ is highly oscillated especially on the domain close to the ultraviolet cutoff, and thus we need to fit our numerical data into some analytical forms and then calculate in Minkowski space. However, the heavy quark mass offers the numerical stability and plausibility which make people to be able to directly calculate the moments from the numerical data without fitting procedure. The results show stability against the choices of the cut-off. We then obtain the moments shown in Table. 2.

Tableau 2. Mesons' moments with $D \omega=(0.87 \mathrm{GeV})^{3}$ (left panel) and $D \omega=(0.55 \mathrm{GeV})^{3}$ (right panel)

\begin{tabular}{|c|c|c|c|c|c|c|c|}
\hline \multicolumn{2}{|c|}{$<(2 x-1)^{m}>$} & $\mathrm{m}=2$ & 4 & $<(2$ & $x-1)^{m}>$ & $\mathrm{m}=2$ & 4 \\
\hline \multirow[t]{2}{*}{$J / \Psi$} & $\perp$ & 0.0260 & 0.0020 & $J / \Psi$ & $\perp$ & 0.0276 & 0.0023 \\
\hline & $\|$ & 0.0049 & $7.23 \times 10^{-5}$ & & $\|$ & 0.0223 & 0.0015 \\
\hline \multirow[t]{2}{*}{$\Upsilon$} & $\perp$ & 0.0192 & 0.0011 & $\Upsilon$ & $\perp$ & 0.0222 & 0.0015 \\
\hline & $\|$ & 0.0028 & $2.37 \times 10^{-5}$ & & $\|$ & 0.0165 & $8.17 \times 10^{-4}$ \\
\hline
\end{tabular}

Tableau 3. Fitting parameters for PDAs with $D \omega=(0.87 \mathrm{GeV})^{3}$ (left panel) and $D \omega=(0.55 \mathrm{GeV})^{3}$ (right panel)

\begin{tabular}{|c|c|c|c|c|c|c|c|}
\hline & & $\mathrm{a}$ & c & & & $\mathrm{a}$ & $\mathrm{c}$ \\
\hline \multirow[t]{2}{*}{$J / \Psi$} & $\perp$ & 3.30 & 12.13 & $J / \Psi$ & $\perp$ & 3.20 & 11.34 \\
\hline & $\|$ & 7.59 & 67.23 & & $\|$ & 3.56 & 14.24 \\
\hline \multirow[t]{2}{*}{$\Upsilon$} & $\perp$ & 3.84 & 16.65 & $\Upsilon$ & $\perp$ & 3.57 & 14.28 \\
\hline & $\|$ & 10.04 & 118.0 & & $\|$ & 4.14 & 19.48 \\
\hline
\end{tabular}

We then fit the PDAs with Eq. (2), and show the obtained parameters in Table. 3. As the parameter $c$ tends to be zero, the PDA becomes the asymptotic form, on the contrary, if $c$ becomes infinite the PDA becomes $\delta$ function. All of the heavy flavour mesons' PDAs are narrower than the asymptotic form, while in light quark case, the PDAs are always broader than the asymptotic form. As the quark mass increases, the shape of the PDAs tends to be narrower, and it can be expected to be a $\delta$ function as the quark mass goes to infinity. For the same quark, the longitudinal PDA in vector mesons is narrower than the transversal PDA. However, the PDAs for $J / \Psi$ and $\Upsilon$ meson still deviate from $\delta$ function. We also compare the results of different coupling strengths. The transversal PDA for the vector meson barely changes as the coupling strength changes, however, the longitudinal PDA gets closer to the transversal part as the interaction strength decreases.

In summary, we compute the heavy vector mesons' PDAs. The PDAs are all narrower than the asymptotic form and as the quark mass increases the form would be closer to $\delta$ function. However, the PDAs still deviate from $\delta$ function. We also compare the effects of different coupling constants. We find that as the coupling constant decreases, the longitudinal and transversal PDAs of vector meson get closer to each other. 


\section{Acknowledgments}

The work was supported by the National Natural Science Foundation of China under Contracts No. 11435001 and No. 11175004 ; the National Key Basic Research Program of China under Contract No. G2013CB834400 ; the U.S. Department of Energy, Office of Nuclear Physics, Contract No. DEAC02-06CH11357.

\section{References}

[1] P. Ball, V.M. Braun, Phys. Rev. D54, 2182 (1996), hep-ph/9602323

[2] G.P. Lepage, S.J. Brodsky, Phys. Lett. B87, 359 (1979)

[3] G.R. Farrar, D.R. Jackson, Phys.Rev.Lett. 43, 246 (1979)

[4] M. Beneke, G. Buchalla, M. Neubert, C.T. Sachrajda, Phys. Rev. Lett. 83, 1914 (1999), hep-ph/9905312

[5] M. Beneke, G. Buchalla, M. Neubert, C.T. Sachrajda, Nucl. Phys. B591, 313 (2000), hep-ph/0006124

[6] J. Forshaw, R. Sandapen, JHEP 1011, 037 (2010), 1007. 1990

[7] B. Cox, J. Forshaw, R. Sandapen, JHEP 0906, 034 (2009), 0905.0102

[8] S. Chekanov, M. Derrick, S. Magill, B. Musgrave, D. Nicholass, J. Repond, R. Yoshida, M. Mattingly, M. Jechow, N. Pavel et al., PMC Phys. A 356, 601 (2007)

[9] F. Aaron, M.A. Martin, C. Alexa, V. Andreev, B. Antunovic, A. Asmone, S. Backovic, A. Baghdasaryan, E. Barrelet, W. Bartel et al., JHEP 2010, 1 (2010)

[10] C. Adloff, V. Andreev, B. Andrieu, V. Arkadov, A. Astvatsatourov, I. Ayyaz, A. Babaev, J. Bähr, P. Baranov, E. Barrelet et al., Phys. Lett. B 483, 23 (2000)

[11] L. Chang, I. Cloet, J. Cobos-Martinez, C. Roberts, S. Schmidt et al., Phys. Rev. Lett. 110, 132001 (2013), 1301.0324

[12] F. Gao, L. Chang, Y.X. Liu, C.D. Roberts, S.M. Schmidt, Phys. Rev. D90, 014011 (2014), 1405.0289

[13] I. Cloët, L. Chang, C. Roberts, S. Schmidt, P. Tandy, Phys. Rev. Lett. 111, 092001 (2013), 1306.2645

[14] L. Chang, C. Mezrag, H. Moutarde, C.D. Roberts, J. Rodríguez-Quintero et al., Phys. Lett. B737, 23 (2014), 1406.5450

[15] L. Chang, I. Cloët, C. Roberts, S. Schmidt, P. Tandy, Phys. rev. lett. 111, 141802 (2013)

[16] G.P. Lepage, S.J. Brodsky, Phys. Rev. D22, 2157 (1980)

[17] I.C. Cloet, C.D. Roberts, Prog. Part. Nucl. Phys. 77, 1 (2014), 1310. 2651

[18] P. Maris, C.D. Roberts, Int. J. Mod. Phys. E12, 297 (2003), nucl-th/0301049

[19] A. Bashir, L. Chang, I.C. Cloet, B. El-Bennich, Y.X. Liu et al., Commun. Theor. Phys. 58, 79 (2012), 1201.3366

[20] S.x. Qin, L. Chang, Y.x. Liu, C.D. Roberts, D.J. Wilson, Phys. Rev. C84, 042202 (2011), 1108.0603

[21] H.Y. Cheng, K.C. Yang, Phys. Rev. D 63, 074011 (2001)

[22] J. Chay, C. Kim, arXiv preprint hep-ph/0009244 (2000) 